\title{
Electron beam energy spread measurements using optical klystron radiation
}

\author{
B. Jia, ${ }^{1,2, *}$ J. Li, ${ }^{1,2}$ S. Huang, ${ }^{3}$ Scott C. Schmidler, ${ }^{4}$ and Y. K. Wu ${ }^{1,2, \dagger}$ \\ ${ }^{1}$ Department of Physics, Duke University, Durham, North Carolina 27708, USA \\ ${ }^{2}$ DFELL, Triangle Universities Nuclear Laboratory, Durham, North Carolina 27708, USA \\ ${ }^{3}$ IHIP, School of Physics, Peking University, Beijing 100871, China \\ ${ }^{4}$ Department of Statistical Science, Duke University, Durham, North Carolina 27708, USA
}

(Received 28 May 2010; published 4 August 2010)

\begin{abstract}
In accelerators, the electron beam longitudinal dynamics critically depend on the energy distribution of the beam. Noninvasive, highly accurate measurement of the energy spread of the electron beam in the storage ring remains a challenge. Conventional techniques are limited to measuring a relatively large energy spread using the energy spread induced broadening effect of radiation source size or radiation spectrum. In this work, we report a versatile method to accurately measure the electron beam relative energy spread from $10^{-4}$ to $10^{-2}$ using the optical klystron radiation. A novel numerical method based on the Gauss-Hermite expansion has been developed to treat both spectral broadening and modulation on an equal footing. A large dynamic range of the measurement is realized by properly configuring the optical klystron. In addition, a model-based scheme has been developed for the first time to compensate the beamemittance-induced inhomogeneous spectral broadening effect to improve the accuracy of the energy spread measurement. Using this technique, we have successfully measured the relative energy spread of the electron beam in the Duke storage ring from $6 \times 10^{-4}$ to $6 \times 10^{-3}$ with an overall uncertainty of less than $5 \%$. The optical klystron is a powerful diagnostic for highly accurate energy spread measurement for storage rings and other advanced electron accelerators.
\end{abstract}

DOI: 10.1103/PhysRevSTAB.13.080702

PACS numbers: 29.27. $-\mathrm{a}, 29.20 . \mathrm{db}, 41.60 . \mathrm{Cr}$

\section{INTRODUCTION}

In electron accelerators, the energy distribution of the electron beam is an essential parameter for the study of the longitudinal beam dynamics and beam instabilities. In the storage ring, a critical indicator of the microwave instability is the increase of the energy spread of the bunched electron beam. For the storage ring free-electron laser (FEL), the induced energy spread determines the FEL power [1-3]. In a storage ring FEL based Compton gamma-ray source [4], the electron beam energy spread determines the minimal energy spread of a collimated Compton gamma beam. For accelerator research, it remains a challenge to measure the electron beam energy spread across a wide range and with a high degree of accuracy.

For the storage ring research, a noninvasive energy spread measurement technique is essential. Such a technique is typically based upon the synchrotron radiation of an electron beam in a static magnetic device as the radiated photon beam carries the information of the electron beam energy spread. This information can be found in the effective radiation source size which is widened due to the energy-dependent trajectories of electrons in a dispersive region. A conventional technique measures the transverse electron beam size at a dispersive location of the storage ring. The effective horizontal beam size, $\sigma_{x}^{\text {eff }}=$

\footnotetext{
*botaojia@fel.duke.edu

†wu@fel.duke.edu
}

$\sqrt{\beta_{x} \epsilon_{x}+\left(\eta_{x} \sigma_{E}\right)^{2}}$ [5], depends on the electron beam emittance $\epsilon_{x}$, local beta function $\beta_{x}$ and eta function $\eta_{x}$, and relative electron beam energy spread $\sigma_{E}$. In this method, the measurement of the energy spread requires the knowledge of beam emittance, and beta function and eta function at the radiation point. These beam parameters are difficult to measure with a high degree of accuracy. Furthermore, this technique cannot be used to measure the electron beam energy spread if the source point eta function $\left(\eta_{x}\right)$ is so small that the energy spread induced beam size broadening is insignificant compared to the true beam size due to the beam emittance, i.e., if $\left(\eta_{x} \sigma_{E}\right) \ll \sqrt{\beta_{x} \epsilon_{x}}$.

Undulator radiation has been used to measure the electron beam energy spread. At ESRF, the energy spread was determined using the broadening of the undulator high harmonic radiation to take advantage of a narrowed undulator spectrum at a higher harmonic [6]. In this scheme, the reduced opening angle of the harmonic radiation beam also increased the sensitivity of the spectrum to the angular spread of the electron beam, leading to the necessity of performing emittance correction. In this method, a good knowledge of beam parameters, such as beta functions in the undulator and beam emittance, are important. At Advanced Photon Source (APS), an angle-integrated spectrum around the fundamental harmonic is used to measure the energy spread [7]. This method has the advantage of not being sensitive to the emittance effect. However, the treatment of the undulator linewidth using a quadrature subtraction introduces a relatively large error [8]. In addition, the required large opening angle compared to the 
natural opening angle of a relativistic beam, available to APS at $7 \mathrm{GeV}$, may not be available for lower energy storage rings with tight vacuum chambers. Furthermore, with high undulator fields, the off-axis harmonic content can corrupt the fundamental spectrum. In fact, the APS method is analogous to the technique of measuring the electron beam energy spread using the measured Compton x-ray or gamma-ray beam spectrum $[9,10]$. Both of these techniques used at ESRF and APS are not suitable for measuring a very small energy spread when the spectrum broadening is small.

The conventional techniques described above, based upon the broadening of either the source size or spectrum of the synchrotron radiation beam, are useful for a relatively large energy spread, but not for a small energy spread. Recognizing that a monoenergetic beam can produce a radiation spectrum with rich modulation using optical interference, an alternative technique can be devised to be sensitive to the small energy spread. Such a measurement technique was developed about two decades ago using the radiation spectrum from an optical klystron $(\mathrm{OK})$ as part of the storage ring FEL research effort with the VEPP-3 storage ring at BINP, Novosibirsk, Russia $[2,11,12]$ and ACO storage ring at Orsay, France [13]. An optical klystron is a magnetic device which consists of two identical undulators sandwiching a buncher magnet. The buncher provides the optical phase delay essential to the formation of the intensity modulation in the optical klystron spectrum. The spectral modulation is reduced due to a finite energy spread of the electron beam; the amount of modulation reduction can be used to determine the energy spread. Assuming a very small energy spread, the previous works [2,11-13] used an analytic formula to describe the optical klystron spectrum. However, this formula only treats the spectral modulation while discarding the spectral broadening. The usefulness of this analytic spectrum formula is limited as the undulator spectral broadening is typically presented in many practical cases.

This work carries out an in-depth and extensive investigation of using the optical klystron radiation to measure the electron beam energy spread. We have developed a novel fitting method based upon Gauss-Hermite expansions to treat the spectral modulation and broadening on an equal footing. By properly configuring the optical klystron, it allows us to determine the energy spread of the electron beam in a wide range and with a good accuracy throughout the range. We have also studied the effect on the radiation spectrum due to the angular distribution of the electron beam, the so-called emittance effect. For the first time, the emittance effect is compensated for the measured spectra using a simulation model to improve the accuracy of the energy spread measurement. This new technique has been used to measure the energy spread of the electron beam in the Duke storage ring. The Duke storage ring is a dedicated circular accelerator for driving several storage ring FELs [14] and a Compton gamma-ray source, the high intensity gamma-ray source [4].

In Sec. II, we revisit the analytic formula which describes the radiation spectrum of the optical klystron under the assumption of small energy spreads. In Sec. III, a novel model is presented to describe the optical klystron spectrum using the Gauss-Hermite expansion, followed by the comparison of these two methods for the cases of a small energy spread. In Sec. IV, the Gaussian-Hermite method is extended to the cases of a larger energy spread when the undulator spectrum is considerable broadened and modulation in the spectrum is no longer well pronounced. Using simulated spectra, the inhomogeneous broadening of the optical klystron spectra due to electron beam emittance is studied in Sec. V. A model based emittance compensation scheme is developed therein to improve the accuracy of the energy spread measurement. Finally, in Sec. VI, we present our experimental results on energy spread measurements using the optical klystron radiation with the electron beam energy spread manipulated in a wide range by tuning the FEL interaction.

\section{A REVIEW OF THE ANALYTIC FORMULA FOR OPTICAL KLYSTRON SPECTRA}

In the storage ring FEL, an optical klystron is typically used to boost the FEL gain by enhancing microbunching in the electron beam. The spontaneous radiation spectrum of an optical klystron is the interference pattern of two optical wave packets emitted by individual electrons passing through two undulators in sequence. The optical phase difference of the two wave packets is controllable using the buncher magnet. The buncher can be set to optimize the spectral modulation and to facilitate spectrum fitting in determining the energy spread of the electron beam. This works well for cases of small energy spreads in which the spectral features of the optical klystron are dominated by the intensity modulation with negligible spectral broadening. A high buncher magnet setting can dramatically increase the richness of spectral modulation, leading to increased sensitivity to the energy spread.

Traditionally, an analytical formula for the optical klystron spectra has been used as a fitting model to find the electron energy spread $[2,11-13,15]$. In this model, the spectra envelop broadening of one undulator is neglected and the modulation effect due to the optical phase delay of two wave packets dominates the optical klystron spectra. Analytically, the modulated spectra of an optical klystron consisting of two planar undulators is expressed as

$$
\begin{aligned}
I(\lambda)= & I_{0}(\lambda)\left[\frac{\sin \left[\pi N_{u}\left(\frac{\lambda_{0}}{\lambda}-1\right)\right]}{\pi N_{u}\left(\frac{\lambda_{0}}{\lambda}-1\right)}\right]^{2} \\
& \times\left\{1+M \cos \left[2 \pi\left(N_{u}+N_{d}\right) \frac{\lambda_{0}}{\lambda}\right]\right\}
\end{aligned}
$$




$$
\begin{gathered}
M=\exp \left\{-\frac{1}{2}\left[4 \pi\left(N_{u}+N_{d}\right) \sigma_{E}\right]^{2}\right\} \\
\lambda_{0}=\frac{\lambda_{u}}{2 \gamma_{0}^{2}}\left(1+\frac{K_{u}^{2}}{2}\right)
\end{gathered}
$$

where $I(\lambda)$ is the spectra intensity at radiation wavelength $\lambda, I_{0}(\lambda) \propto 1 / \lambda^{4}$ is the amplitude factor, $\lambda_{0}$ is the resonant wavelength, $K_{u}=\left(e B_{0} \lambda_{u}\right) /(2 \pi m c)$ is the dimensionless undulator parameter, $e$ and $m$ are the charge and mass of the electron, $c$ is the speed of light in vacuum, $B_{0}$ is the peak magnetic field, $\gamma_{0}=E /\left(m c^{2}\right)$ is the relativistic parameter for the mean electron energy, $E$ is the electron energy, $N_{d}$ is the delayed number of optical cycles of the second undulator radiation controlled by the buncher setting, $N_{u}$ is the number of undulator periods, $M$ is the modulation factor, and $\sigma_{E}$ is the relative electron beam energy spread. By fitting this analytic formula to experimentally measured spectra, $\sigma_{E}$ can be determined. However, this formula was derived for a small energy spread $\left(\sigma_{E} \ll \frac{1}{N_{u}}\right)$, and has limited applicability for studying the effect of energy spread increase due to a high bunch current in a storage ring or due to FEL interaction in a storage ring FEL. To overcome this difficulty, we have developed a new fitting model which is suitable for a wide range of energy spread values. In the following sections, we refer to formula Eq. (1) as the small energy spread (SES) approximation method.

\section{A NEW MODEL FOR OPTICAL KLYSTRON SPECTRA}

\section{A. Gauss-Hermite quadrature expansion for spontaneous radiation spectrum}

In this and the following sections, we study the impact of electron beam energy spread on the optical klystron spectrum. An electron beam with a very small emittance is used in this section so that spectrum broadening due to the angular spread of the electron beam can be neglected.

The on-axis spontaneous radiation intensity as a function of radiation wavelength $\lambda$ for one electron passing through an OK is given by $[13,16]$

$$
\begin{aligned}
I(\lambda)= & I_{1}(\lambda, \gamma)\left[\frac{\sin \left[\pi N_{u}\left(\frac{\lambda_{r}(0)}{\lambda}-1\right)\right]}{\pi N_{u}\left(\frac{\lambda_{r}(0)}{\lambda}-1\right)}\right]^{2} \\
& \times\left\{1+\cos \left[2 \pi\left(N_{u}+N_{d}\right) \frac{\lambda_{r}(0)}{\lambda}\right]\right\}, \\
& \lambda_{r}(\theta)=\frac{\lambda_{u}}{2 \gamma^{2}}\left(1+\frac{K_{u}^{2}}{2}+\gamma^{2} \theta^{2}\right),
\end{aligned}
$$

where $I_{1}(\lambda, \gamma) \propto 1 /\left(\lambda^{4} \gamma^{2}\right)$ is the amplitude factor, $\lambda_{r}(\theta)$ is the resonant radiation wavelength as a function of the offaxis angle $\theta$, and $\gamma$ is the relativistic parameter for individual electron energy. For an electron beam, the effect due to inhomogeneous electron energy should be taken into account. The resulting spectrum of a collection of individual electrons with various energies is broader than that of a single electron. The measured spectrum of an electron beam is the integral of Eq. (4) over all possible electron energies. Generally, for an electron beam the relativistic electron energy $\gamma$ is assumed to follow a Gaussian distribution with mean $\gamma_{0}$ and standard deviation $\sigma_{\gamma}$. Taking into account the measurement uncertainty, the spectrum intensity can be modeled by the expression

$$
\begin{aligned}
I_{\text {total }}(\lambda)= & \int_{-\infty}^{\infty} I_{1}(\lambda, \gamma)\left[\frac{\sin \left[\pi N_{u}\left(\frac{\lambda_{r}(0)}{\lambda}-1\right)\right]}{\pi N_{u}\left(\frac{\lambda_{r}(0)}{\lambda}-1\right)}\right]^{2} \\
& \times\left\{1+\cos \left[2 \pi\left(N_{u}+N_{d}\right) \frac{\lambda_{r}(0)}{\lambda}\right]\right\} \\
& \times f_{\gamma}\left(\gamma_{0}, \sigma_{\gamma}\right) d \gamma+h+\xi, \\
f_{\gamma}\left(\gamma_{0}, \sigma_{\gamma}\right)= & \frac{1}{\sqrt{2 \pi} \sigma_{\gamma}} \exp \left(-\frac{\left(\gamma-\gamma_{0}\right)^{2}}{2 \sigma_{\gamma}^{2}}\right),
\end{aligned}
$$

where $I_{\text {total }}$ is the measured spectrum intensity at $\lambda, h$ is the average measurement signal background, and $\xi \sim$ $N\left(0, \sigma_{\xi}\right)$ is the measurement noise which is assumed to be normally distributed with standard deviation $\sigma_{\xi}$. Among all quantities, the energy spread

$$
\sigma_{E}=\frac{\sigma_{\gamma}}{\gamma_{0}}
$$

is of special interest and the final goal is to determine the energy spread from a measured spectrum using Eq. (6). As can be seen, while $\gamma$ for individual electrons cannot be directly measured, it is integrated out in Eq. (6) with respect to a density distribution function of $\gamma$.

Next, we discuss the fitting method based on this model.

The integral in Eq. (6) does not have a straightforward analytic solution. We have developed several methods [17] to solve the challenging problem of fitting the energy spread, including Bayesian and nonlinear least-squares methods using Monte Carlo and Gauss-Hermite quadrature integral approximations. In this paper, we present the Gauss-Hermite expansion which is highly efficient while providing good accuracy. In the remainder of this paper, we refer to this as the G-H method.

Gauss-Hermite quadrature $[18,19]$ is an extension of the Gauss quadrature method for approximating of a particular type of univariate integral:

$$
\int_{-\infty}^{\infty} \exp \left(-t^{2}\right) g(t) d t \approx \sum_{i=1}^{n} \omega_{i, n} g\left(t_{i, n}\right)
$$

where

$$
\omega_{i, n}=\frac{2^{n-1} n ! \sqrt{\pi}}{n^{2}\left[H_{n-1}\left(t_{i, n}\right)\right]^{2}},
$$

and $t_{i, n}$ is the $i$ th zero of the $n$th order Hermite polynomial $H_{n}(t), H_{n}\left(t_{i, n}\right)=0$. The remainder term of the expansion 
(7) has the form

$$
R_{n}=\frac{n ! \sqrt{\pi}}{2^{n}(2 n) !} g^{(2 n)}(t)
$$

where $g^{(2 n)}(t)$ is the $2 n$th order derivative of $g(t)$. As $n$ increases, this remainder term converges to zero quickly.

Using Eq. (7), the optical klystron spectrum can be expanded as

$$
\begin{aligned}
I_{\text {total }}(\lambda)= & I_{A} \sum_{i=1}^{n} \frac{2^{n-1} n !}{n^{2}\left[H_{n-1}\left(t_{i, n}\right)\right]^{2}} \cdot \frac{1}{\lambda^{4}\left(\gamma_{0}+\sqrt{2} \sigma_{\gamma} t_{i, n}\right)^{2}} \\
& \times\left\{\frac{\sin \left[\pi N_{u}\left(\frac{\lambda_{u}\left(1+K_{u}^{2} / 2\right)}{2 \lambda\left(\gamma_{0}+\sqrt{2} \sigma_{\gamma} t_{i, n}\right)^{2}}-1\right)\right]}{\pi N_{u}\left(\frac{\lambda_{u}\left(1+K_{u}^{2} / 2\right)}{2 \lambda\left(\gamma_{0}+\sqrt{2} \sigma_{\gamma} t_{i, n}\right)^{2}}-1\right)}\right\}^{2} \\
& \times\left\{1+\cos \left[\pi\left(N_{u}+N_{d}\right) \frac{\lambda_{u}\left(1+K_{u}^{2} / 2\right)}{\lambda\left(\gamma_{0}+\sqrt{2} \sigma_{\gamma} t_{i, n}\right)^{2}}\right]\right\} \\
& +R_{n}+h+\xi,
\end{aligned}
$$

where $I_{A}$ is the amplitude normalization factor. With properly chosen $n$, higher order terms are very small and can be safely neglected. The parameters can now be determined using nonlinear least-squares fitting. In the following, we study the goodness of fit, and the spectrum broadening effect due to the energy spread, using simulated spectra. We compare fitting results using the G-H method to those using the SES method.

\section{B. G-H method vs SES method}

In this section, we evaluate and compare two methods to determine the energy spread, the SES method [Eq. (1)], and the G-H method [Eq. (10)]. The comparison will be carried out using simulated optical klystron spectra with known energy spread values. The scheme to generate the simulated spectra is described in detail in Sec. V. The energy spread is estimated using least-squares fitting with either the SES or the G-H method. The fitted value of the energy spread is compared to the known value as it is varied. To focus on the effect of the energy spread, the simulated spectra are generated using an electron beam with a very small emittance and are collected close to the axis of radiation through a small aperture. Care has been taken to make sure that the impact of electron emittance and finite collection angle on the spectra broadening is negligible. The relative energy spread set value in the simulation is denoted by $\sigma_{\mathrm{E}}^{\text {set }}$, and the corresponding fitted value by $\sigma_{\mathrm{E}}^{\mathrm{fit}}$. When it is necessary to distinguish the fitted values of the two methods, we will explicitly use $\sigma_{\mathrm{E}, \mathrm{GH}}^{\mathrm{fit}}$ and $\sigma_{\mathrm{E}, \mathrm{SES}}^{\mathrm{fit}}$ to denote the value estimated using the G-H and SES methods, respectively.

Figures 1 and 2 show the simulated and fitted spectra with $N_{d}=31 . N_{d}=31$ is chosen for simulations since modulation is deep enough and this value is practical in the

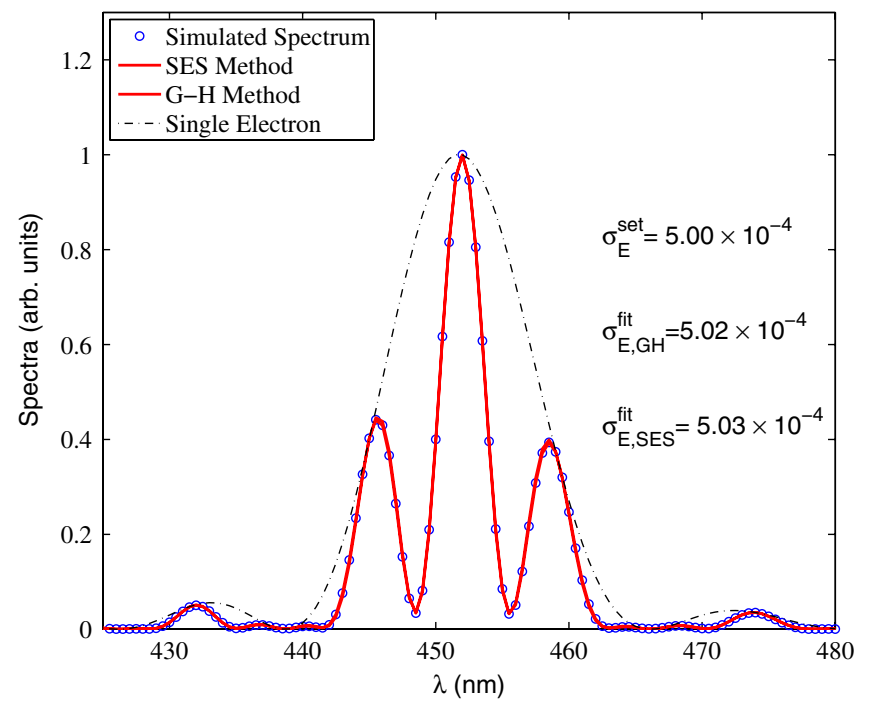

FIG. 1. (Color) Simulated optical klystron spectrum and fitted curves with both $\mathrm{G}-\mathrm{H}$ and SES methods. The simulated spectrum is generated with $N_{d}=31, \sigma_{\mathrm{E}}^{\text {set }}=5.00 \times 10^{-4}$, and $\epsilon_{x}=1 \mathrm{~nm}$; the other beam parameters are listed in Table I. The fitted curve using the G-H method and the fitted curve using the SES method are indistinguishable and both agree well with the simulated spectrum. A single undulator spectrum $\left(N_{u}=33\right)$ with a monoenergetic beam is also shown as an expected envelope of the optical klystron spectrum when the energy spread is small.

experimental setup to avoid unnecessary high buncher currents.

Figure 1 shows a case with small relative energy spread $\sigma_{\mathrm{E}}^{\mathrm{set}}=5.00 \times 10^{-4}$. Fitted curves using SES and G-H

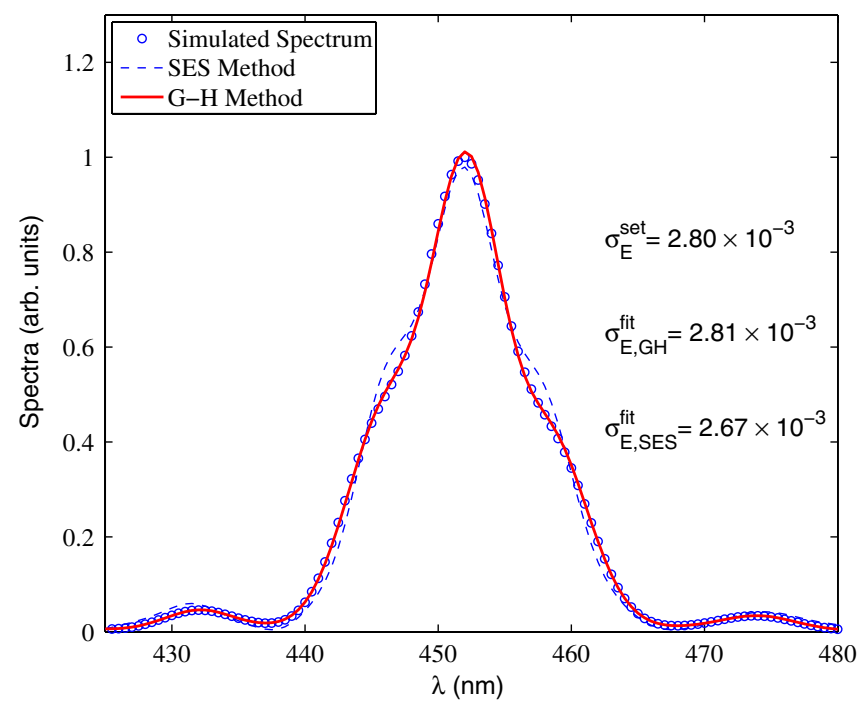

FIG. 2. (Color) A simulated spectrum and related fitted curves. The simulated spectrum is generated with $\sigma_{\mathrm{E}}^{\text {set }}=2.80 \times 10^{-3}$, $\epsilon_{x}=1 \mathrm{~nm}$, and $N_{d}=31$. The red solid curve is a fitted spectrum using the G-H method; the black dashed curve is the fitted spectrum using the SES method. The fitted values of the energy spread using both methods are listed in the plot. 
methods overlap, and are indistinguishable from each other in the plot. The fitted energy spread values differ from $\sigma_{\mathrm{E}}^{\text {set }}$ by less than $1 \%$ : $\sigma_{\mathrm{E}, \mathrm{fES}}^{\mathrm{fit}}=5.03 \times 10^{-4}$ and $\sigma_{\mathrm{E}, \mathrm{GH}}^{\mathrm{fit}}=$ $5.02 \times 10^{-4}$. As $\sigma_{\mathrm{E}}^{\text {set }}$ is increased to larger values, e.g. $2.80 \times 10^{-3}$, the SES method produces a poor fit (Fig. 2); the fitted spectrum shows significant deviation from the simulated spectrum around the shoulders of the peak. The $\sigma_{\mathrm{E}, \mathrm{fES}}^{\mathrm{fit}}$ is about $5 \%$ lower than $\sigma_{\mathrm{E}}^{\text {set }}$. On the other hand, the G-H method produces a good fit (Fig. 2) and $\sigma_{\mathrm{E}, \mathrm{GH}}^{\mathrm{fit}}$ differs from $\sigma_{\mathrm{E}}^{\text {set }}$ by about $1 \%$.

The two methods are compared in Fig. 3 for a wide range of energy spread values using simulated spectra. Relative differences,

$$
\Delta \sigma_{\mathrm{E}}^{\mathrm{diff}}=\frac{\sigma_{\mathrm{E}}^{\mathrm{fit}}-\sigma_{\mathrm{E}}^{\mathrm{set}}}{\sigma_{\mathrm{E}}^{\mathrm{set}}},
$$

are also shown for both SES and G-H methods in the inset. As can be seen, the performance of the SES method depends strongly on the value of $\sigma_{\mathrm{E}} \cdot \sigma_{\mathrm{E}, \mathrm{SES}}^{\mathrm{fit}}$ deviates from $\sigma_{\mathrm{E}}^{\text {set }}$ up to $18 \%$. In contrast, the $\mathrm{G}-\mathrm{H}$ method is much more robust and $\sigma_{\mathrm{E}, \mathrm{GH}}^{\mathrm{fit}}$ deviates less than $2.5 \%$ as $\sigma_{\mathrm{E}}^{\text {set }}$ varies from $5.00 \times 10^{-4}$ to $4.50 \times 10^{-3}$. Another observation is that the error bars increase significantly for the SES method as the energy spread becomes larger, making the SES method unsuitable for the region of a large energy spread, in our case, when $\sigma_{\mathrm{E}}>2.5 \times 10^{-3}$. The error bar is the $70 \%$ confidence interval obtained from asymptotic normality assumption. When the energy spread is small, a higher degree of spectrum modulation provides

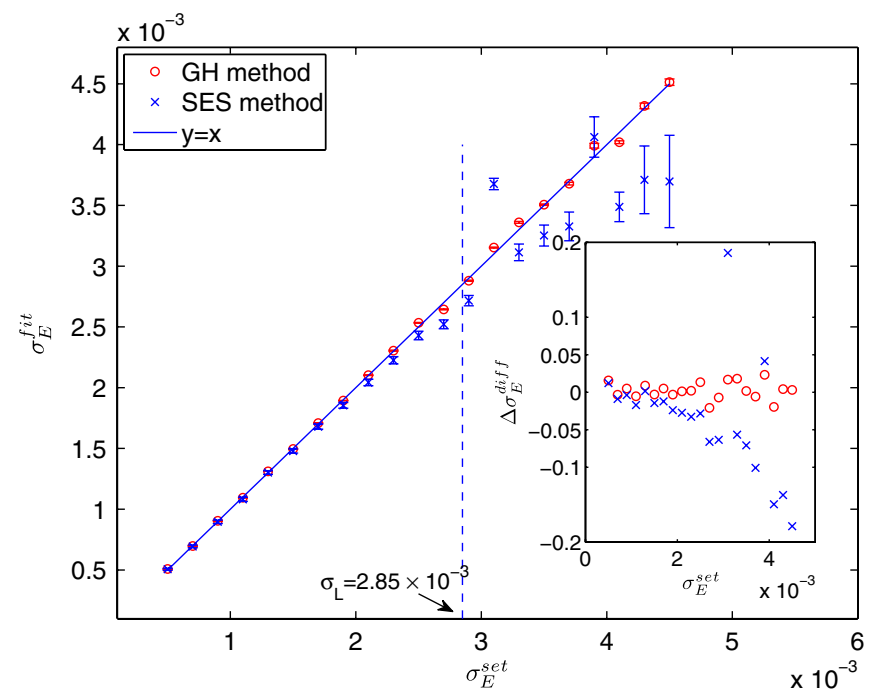

FIG. 3. (Color) Comparison of the fitted energy spread $\sigma_{\mathrm{E}}^{\text {fit }}$ and set energy spread $\sigma_{\mathrm{E}}^{\text {set }}$. Parameters to generate simulated spectra are listed in Table I with $N_{d}=31$ and $\epsilon_{x}=1 \mathrm{~nm}$. The fitted values of the energy spread using the G-H method are plotted as circles, while the fitted values using the SES method are plotted as crosses. The error bars show the RMS uncertainty of the fitting. The inset shows the relative difference between the fitted and set values of the energy spread, $\Delta \sigma_{E}^{\text {diff }}[$ [Eq. (11)]. more constraints to the energy spread fitting. Even in this region, the G-H method provides overall better fitting than the SES method.

When the relative energy spread is varied from $5.00 \times$ $10^{-4}$ to $4.50 \times 10^{-3}$, the RMS statistical fit error using the G-H method is less than $1 \%$. Using this data set, we can define a particular range of the energy spread in which the G-H method is mostly suitable for using an optical klystron with a relatively large $N_{d}$. This energy spread value can be chosen as

$$
\sigma_{L}=\frac{\sigma_{u}}{2}=\frac{0.09405}{N_{u}}
$$

where $\sigma_{u}=\frac{1}{2} \frac{1}{2 \sqrt{2 \ln 2}} \frac{0.8859}{N_{u}}$ is an equivalent RMS energy spread due to undulator radiation modeled using a Gaussian distribution for the single undulator spectrum, a sinc-function. A factor of $1 / 2$ in $\sigma_{u}$ is to account for the impact of the energy spread on the undulator linewidth since the resonance wavelength $\lambda_{r} \propto \gamma^{-2}$. The other factor of $1 / 2$ in $\sigma_{L}$ is included to ensure that the energy spread is not too large to prevent a complete wash-away of spectrum modulation features by the spectrum broadening effect. For our optical klystron system with $N_{u}=33, \sigma_{L}=2.85 \times$ $10^{-3}$. Certainly, the G-H method is still useful for an energy spread somewhat larger than $\sigma_{L}$. However, the robustness of the fitting can be significantly improved if a new configuration of the optical klystron is used with a small buncher setting. This new configuration can extend the usefulness of the G-H method to even larger energy spread values as described in the following section.

\section{OPTICAL KLYSTRON CONFIGURATION FOR LARGER ENERGY SPREAD}

When it is increased above the level of $\sigma_{L}$, the energy spread of the electron beam causes significant broadening of the undulator spectra, washing away the modulation features of the optical klystron. In this scenario, the larger energy spread can be determined using the broadened spectra of a single undulator [7]. The optical klystron system can be easily reconfigured to have a small $N_{d}$. The choice of $N_{d}$ here is to connect the optical phase of two wave packets emitted in two undulators, making the optical klystron similar to a single undulator system with twice the number of periods. This increases the sensitivity of detecting the spectra broadening effect induced by the energy spread. The equivalent RMS energy spread associated with this long "undulator" is $\bar{\sigma}_{u}=\sigma_{u} / 2$.

In this optical klystron configuration with a small buncher setting, the G-H method accurately estimates the energy spread. The robustness of the G-H method is demonstrated here using a case with a relatively large energy spread of $\sigma_{\mathrm{E}}^{\text {set }}=5.00 \times 10^{-3}$ and $N_{d}=1.0$ (Fig. 4). The simulated spectrum is fitted using the G-H method with the same number of modes as for the smaller energy spread cases. It yields a fitted energy spread of $5.01 \times 10^{-3}$, 


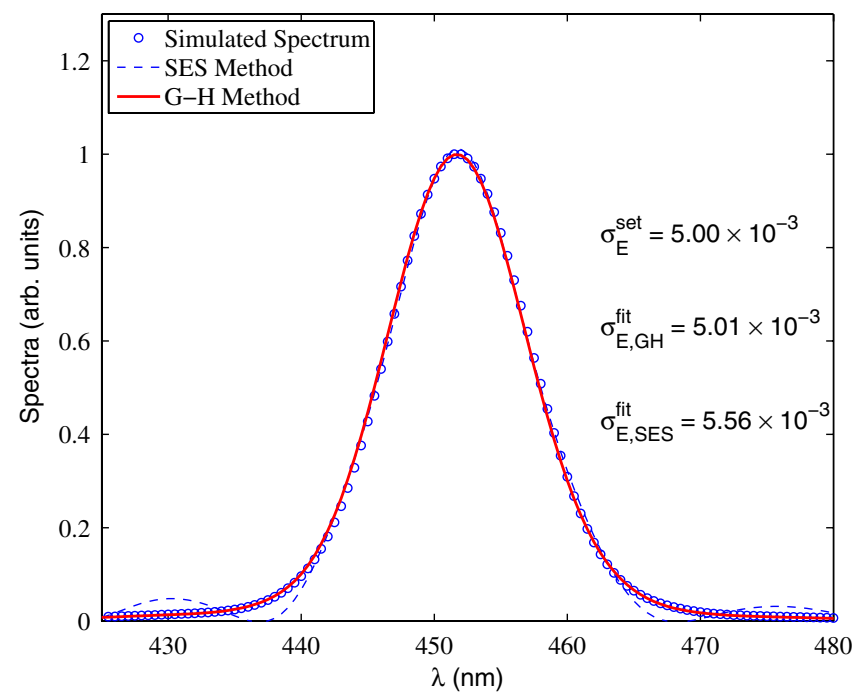

FIG. 4. (Color) A simulated spectrum and related fitted curves. Parameters to generate simulated spectra are listed in Table I with $\sigma_{\mathrm{E}}^{\text {set }}=5.00 \times 10^{-3}, N_{d}=1$, and $\epsilon_{x}=1 \mathrm{~nm}$. The red solid curve is a fitted spectrum using the G-H method; the black dashed curve is the fitted spectrum using the SES method. The fitted values of the energy spread using both methods are listed in the plot.

differing from $\sigma_{\mathrm{E}}^{\text {set }}$ by less than $1 \%$. At this large energy spread, the SES method produces a poor fit to the spectrum and a large discrepancy $(\sim 11 \%)$ in the fitted energy spread value.

Using this small $N_{d}$ configuration of the optical klystron, the G-H method can be used to accurately measure larger values of the energy spread, up to a value $\sigma_{H}$, where

$$
\sigma_{H}=2 \sigma_{L}=\frac{0.1881}{N_{u}}
$$

For our case $\sigma_{H}=5.70 \times 10^{-3}$. Figure 5 shows the comparison between the fitted values and set values, as the energy spread is changed from $2.85 \times 10^{-3}$ to $5.70 \times$ $10^{-3}$. The fitted values deviate from the set values by a maximum of $2.5 \%$. In the region $\sigma_{\mathrm{E}}^{\text {set }}=2.85-5.70 \times 10^{-3}$, the energy spread values are found using two configurations of the optical klystron with $N_{d}=31$ and $N_{d}=1$. When $\sigma_{\mathrm{E}}^{\text {set }}>\sigma_{L}=2.85 \times 10^{-3}$, the $N_{d}=1$ configuration produces somewhat better fitting with a smaller discrepancy from the set value. In general, when the energy spread of the electron beam is between $\sigma_{L}$ and $\sigma_{H}$ (region R2 in Fig. 5), the optical klystron should be configured with a small $N_{d}$ to improve the accuracy of energy spread measurements.

When the energy spread increases beyond $\sigma_{H}$, the modulation features of the spectrum are washed away and the associated parameters $\left(\lambda_{u}, N_{u}, K_{u}, \gamma_{0}\right)$ become unstable to fit. However, in this case $\sigma_{H}=2 \bar{\sigma}_{u}$, the optical klystron spectrum closely resembles a Gaussian function as the spectrum features are mostly determined by the

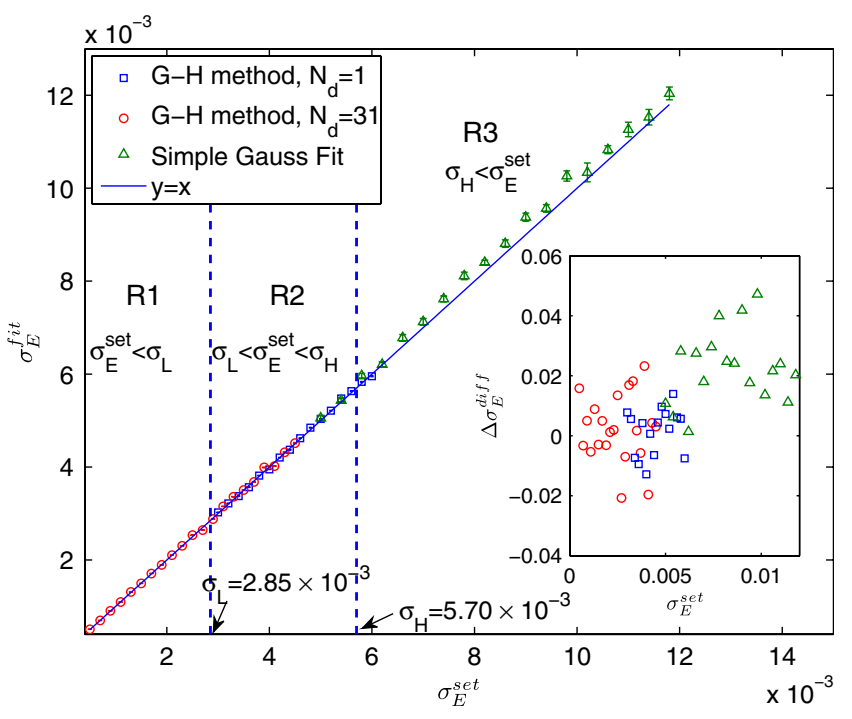

FIG. 5. (Color) Comparison of the fitted and set values of the energy spread in three regions: R1 $\left(\sigma_{\mathrm{E}}^{\text {set }}<\sigma_{L}\right)$, R2 $\left(\sigma_{L}<\sigma_{\mathrm{E}}^{\text {set }}<\right.$ $\left.\sigma_{H}\right)$, and $\mathrm{R} 3\left(\sigma_{\mathrm{E}}^{\text {set }}>\sigma_{H}\right)$. Parameters to generate simulated spectra are listed in Table I with $\epsilon_{x}=1 \mathrm{~nm}$. For small energy spread, $N_{d}=31$, and for large energy spread, $N_{d}=1$. The G-H method is used to fit the spectra in both $\mathrm{R} 1$ and $\mathrm{R} 2$ regions; the simple Gaussian fit is used for some spectra in the region R2 and all the spectra in the region $\mathrm{R} 3$. The inset shows the relative discrepancy between the fitted and set values of the energy spread in the three regions.

electron beam energy spread. In this region ( $\mathrm{R} 3$ in Fig. 5), the energy spread can be found by fitting the spectrum to a Gaussian function and then correcting the width by quadrature subtraction,

$$
\sigma_{\mathrm{E}}=\sqrt{\left(\sigma_{g} / 2\right)^{2}-\bar{\sigma}_{u}^{2}}=\sqrt{\left(\sigma_{g} / 2\right)^{2}-\left(\sigma_{u} / 2\right)^{2}},
$$

where $\sigma_{g}$ is the RMS width of Gaussian function which best fits the spectrum in the least-squares sense. As shown in Fig. 5, this Gaussian-fit method produces reasonably good results in the region R3; the fitted values of the energy spread typically deviate from the set values by $2 \%-5 \%$. However, a simple Gaussian line shape fit in the region R2 produces a fitted spectrum which is typically poorer than that obtained from the G-H method.

It is worth pointing out that the simple Gaussian fit is an approximate limiting case of the G-H method with a single Gauss-Hermite mode and modulation parameters set to fixed values. In fact, in the region $\sigma_{\mathrm{E}}>\sigma_{L}$, better and more reliable fits could be produced by optimizing the number of Gauss-Hermite modes used in the fitting. This requires the development of an adaptive $\mathrm{G}-\mathrm{H}$ method for different ranges of energy spread values. This development, while interesting, falls outside the scope of this work.

In Secs. III and IV, we have demonstrated that optical klystron radiation can be used to accurately determine a wide range of values of the electron beam energy spread by 
applying the G-H method. When the energy spread is small $\left(\sigma_{\mathrm{E}}<\sigma_{L}\right)$, the optical klystron should be configured with a large $N_{d}$ to enhance intensity modulation in the spectrum. With a larger energy spread $\left(\sigma_{\mathrm{E}}>\sigma_{L}\right)$, the optical klystron can be configured with a small $N_{d}\left(N_{d} \sim 1\right)$ forming an equivalent "long" undulator to enhance the ability of detecting spectral broadening due to the energy spread. In this region, the optical klystron spectrum can be fit using either the G-H method or a simple Gaussian fit with the undulator linewidth correction, and choice should be made depending on expected range in which the energy spread will lie.

Up to this point, the impact of the angular spread of the electron beam has been kept small by using an electron beam with a small emittance. In the next section, the spectrum inhomogeneous broadening induced by beam emittance will be studied in detail.

\section{EMITTANCE EFFECT}

In Secs. III and IV, fitting methods were given for various ranges of the electron beam energy spread and demonstrated using simulated spectra of an optical klystron. These spectra are generated for well-collimated photon beams produced by an electron beam with small emittance, where broadening due to the angular spread of the electron beam (the emittance effect) is negligible. We now turn our attention to the emittance effect. We describe a compensation scheme to correct the emittance-related spectral broadening and improve the accuracy of determining the energy spread.

\section{A. Simulation of measured spectra with emittance effect}

We consider an electron moving along the longitudinal ( $z$ axis) direction (Fig. 6) in a beam with a horizontal beam emittance $\epsilon_{x}$ and energy spread $\sigma_{\mathrm{E}}$. A small aperture downstream is used to collect the radiation. The half opening angle of the aperture is denoted by $\theta_{a p}$. The electron momentum makes a horizontal angle with respect to the $z$ axis; this angle can be approximated by the normalized

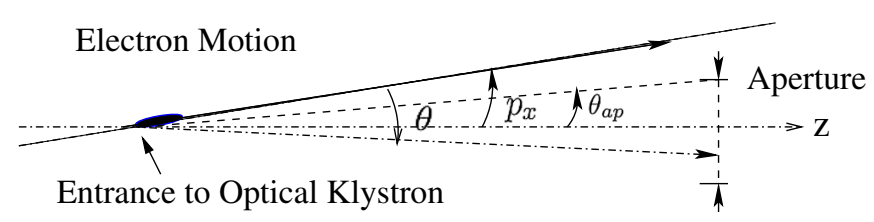

FIG. 6. Illustration of the electron trajectory and emission in an optical klystron with planar undulators. The electron is moving in the midplane of the undulator field with a horizontal angle $p_{x}$ (the normalized horizontal momentum) with respect to the longitudinal $z$ axis. The radiation is collected behind an aperture opening with half-angle $\theta_{a p}$. The radiation is collected at a particular opening angle $\theta$ with respect to the electron trajectory. transverse momentum, $p_{x}$. Radiation is emitted at angle $\theta$ with respect to the electron motion. The following discussion focuses on the horizontal emittance effect, which is much larger than the vertical emittance effect for the Duke storage ring.

The radiation intensity for a single electron is given by

$$
\begin{aligned}
\left|\frac{d^{2} I}{d \lambda d \Omega}\right|= & \frac{e^{2}}{4 \epsilon_{0}} \frac{K_{u}^{2} L_{u}^{2}}{\gamma^{2} \lambda^{4}} \cdot\left[\frac{\sin \left(\pi N_{u} \frac{\lambda_{r}(\theta)-\lambda}{\lambda}\right)}{\pi N_{u} \frac{\lambda_{r}(\theta)-\lambda}{\lambda}}\right]^{2} \\
& \cdot\left(1+\cos \left[2 \pi\left(N_{u}+N_{d}\right) \frac{\lambda_{r}(0)}{\lambda}\right.\right. \\
& \left.\left.+\frac{\pi\left(L_{u}+L_{d}\right)}{\lambda} \theta^{2}\right]\right) \cdot F_{J}(n),
\end{aligned}
$$

where $d \Omega$ is the solid angle, $\frac{\pi\left(L_{u}+L_{d}\right)}{\lambda} \theta^{2}$ is the additional phase difference due to the off-axis optical path advance, $L_{d}$ is the drift length in the buncher section, $L_{u}=N_{u} \lambda_{u}$, $F_{J}(n)$ is the $[J J]$ factor:

$$
F_{J}=\left(\sum_{k=-\infty}^{\infty}(-1)^{k} J_{2 k}(\alpha)\left[J_{k}(\xi)-J_{k+1}(\xi)\right]\right)^{2},
$$

where

$$
\begin{gathered}
\alpha=2 \frac{K_{u} \gamma \theta \cos \phi}{1+\frac{K_{u}^{2}}{2}+\gamma^{2} \theta^{2}}, \\
\xi=\frac{K_{u}^{2}}{1+\frac{K_{u}^{2}}{2}+\gamma^{2} \theta^{2}} .
\end{gathered}
$$

For a very small collection aperture as used in these simulations, the higher harmonic radiation contributes negligibly to the simulated optical klystron spectrum, which is centered around the wavelength of the fundamental radiation.

The radiation spectrum for an electron beam is the superposition of single electron emission with different angles $p_{x}$ and energies $\gamma$. We assume independent normal distributions for $p_{x}$ and $\gamma$, and the measured spontaneous radiation spectrum is

$$
\begin{aligned}
& I_{\text {total }}(\lambda)= \int_{-\infty}^{\infty} d \gamma \int_{-\infty}^{\infty} d p_{x p_{x}-\theta_{a p} \leq \theta \leq p_{x}+\theta_{a p}}\left|\frac{d^{2} I}{d \lambda d \Omega}\right| \\
& \times f_{p_{x}}\left(0, \sigma_{p_{x}}\right) f_{\gamma}\left(\gamma_{0}, \sigma_{\mathrm{E}}^{\text {set }} \gamma_{0}\right) d \Omega, \\
& f_{p_{x}}\left(0, \sigma_{p_{x}}\right)=\frac{1}{\sqrt{2 \pi} \sigma_{p_{x}}} \exp \left(-\frac{p_{x}^{2}}{2 \sigma_{p_{x}}^{2}}\right), \\
& f_{\gamma}\left(\gamma_{0}, \sigma_{\mathrm{E}}^{\text {set }} \gamma_{0}\right)=\frac{1}{\sqrt{2 \pi}\left(\gamma_{0} \sigma_{\mathrm{E}}^{\text {set }}\right)} \exp \left(-\frac{\left(\gamma-\gamma_{0}\right)^{2}}{2\left(\gamma_{0} \sigma_{\mathrm{E}}^{\text {set }}\right)^{2}}\right),
\end{aligned}
$$

where $\sigma_{\mathrm{E}}^{\text {set }}$ and $\sigma_{p_{x}}$ are the set values when calculating the simulated measured spectra, and $\sigma_{p_{x}}$ is given by 


$$
\sigma_{p_{x}}=\sqrt{\epsilon_{x} \gamma_{x}}=\sqrt{\frac{\epsilon_{x}}{\beta_{0 x}}},
$$

where $\gamma_{x}$ is the Twiss parameter at the entrance of the optical klystron and $\beta_{0 x}$ is the beta function at the center of the optical klystron. Equation (19) is used as the model to generate the simulated spectra throughout the paper.

\section{B. Evaluation of the emittance effect}

Both the energy spread and the emittance effect contribute to spectral broadening. Thus the G-H method of Sec. III A, which does not take into account spectral broadening due to emittance, will tend to overestimate the energy spread. Table I gives a set of electron beam parameters for generating simulated spectra, chosen to match those used in the experiments of Sec. VI. The G-H method was used to fit the simulated spectra and determine $\sigma_{\mathrm{E}}^{\text {fit }}$.

When emittance is small (e.g. $\epsilon_{x}=1 \mathrm{~nm}$ ), the G-H method estimates the energy spread accurately as shown in Fig. 3. When the emittance is large, as shown in Fig. 7 with $\epsilon_{x}=50 \mathrm{~nm}, \sigma_{\mathrm{E}}^{\text {fit }}$ can deviate from $\sigma_{\mathrm{E}}^{\text {set }}$ noticeably when $\sigma_{\mathrm{E}}^{\text {set }}<3 \times 10^{-3}$. In these cases, correction for the emittance effect is desirable.

Note that electron energy inhomogeneity and angular inhomogeneity are two competing effects. This can be observed in Fig. 7, where with certain combinations of emittance and energy spread, the emittance effect begins to impact the measured spectra. To describe these two competing spectral broadening effects, we define a "scaled emittance" $a_{x}$ as the ratio of the two effects:

$$
a_{x}=\frac{\left(\gamma \sigma_{p_{x}}\right)^{2}}{\sigma_{\mathrm{E}}^{\text {set }}}=\frac{\gamma^{2} \epsilon_{x}}{\sigma_{\mathrm{E}}^{\text {set }} \beta_{0 x}} .
$$

TABLE I. Electron beam and OK parameters for generating simulated spectra.

\begin{tabular}{lc}
\hline \hline Electron beam parameters & Value \\
\hline Mean energy $(\mathrm{MeV})$ & 400.0 \\
Mean energy $\left(\gamma_{0}\right)$ & 782.8 \\
Energy spread $\sigma_{\mathrm{E}}^{\text {set }}\left(\times 10^{-3}\right)$ & {$[0.4,12]$} \\
Horizontal emittance $\epsilon_{x}(\mathrm{~nm})$ & {$[1,50]$} \\
$\beta$ function at center of optical klystron, $\beta_{0, x}(\mathrm{~m})$ & 4.5 \\
Horizontal angular spread $\sigma_{p x}$ & $\sqrt{\frac{\epsilon_{x}}{\beta_{0 x}}}$ \\
\hline Optical klystron parameters & Value \\
\hline RMS undulator parameter $K_{u}$ & 3.013 \\
Undulator period $\lambda_{u}(\mathrm{~m})$ & 0.100 \\
Number of periods for each undulator $N_{u}$ & 33 \\
Dispersion parameter $N_{d}$ & 31 or 1 \\
Drift length of buncher section $L_{d}(\mathrm{~m})$ & 0.82 \\
Resonant wavelength $\lambda_{s}(\mathrm{~nm})$ & 452.0 \\
Spectrometer aperture angle $\theta_{a p}(\mathrm{rad})$ & $2.7 \times 10^{-5}$ \\
\hline \hline
\end{tabular}

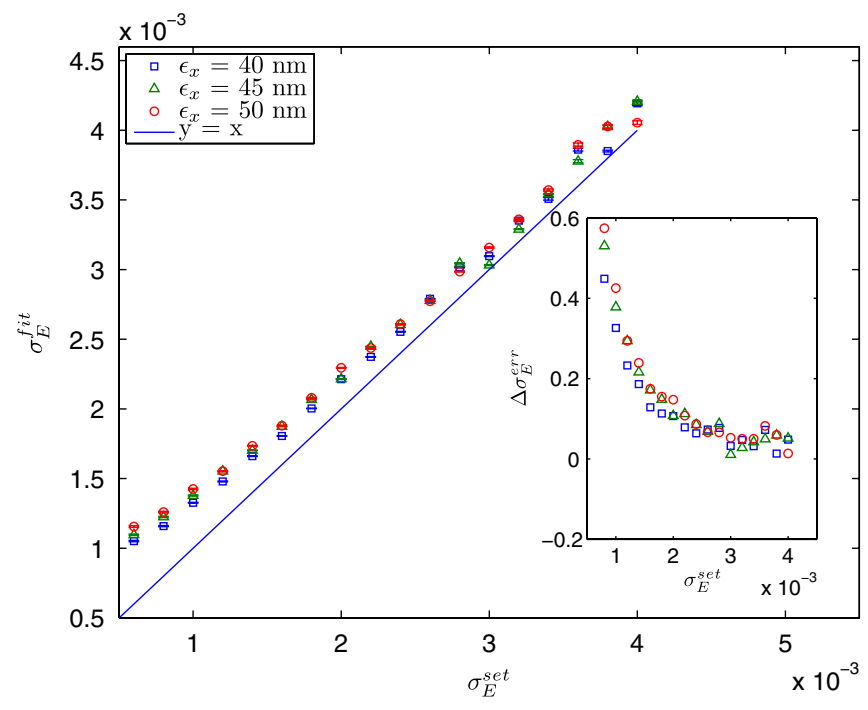

FIG. 7. (Color) Comparison of the fitted and set values of the energy spread with large emittance values, $\epsilon_{x}=40,45,50 \mathrm{~nm}$. Other simulation parameters are found in Table I with $N_{d}=31$. The inset shows the relative difference between the fitted and set values, $\sigma_{\mathrm{E}}^{\text {diff }}[$ Eq. (11)].

\section{Emittance correction}

When the emittance effect is large enough, a correction to the fitted energy spread is desirable. We introduce $\sigma_{\mathrm{E}, \boldsymbol{\epsilon}_{x}}$ to describe the correction needed due to emittance effect,

$$
\sigma_{\mathrm{E}, \boldsymbol{\epsilon}_{x}}=\sqrt{\left(\sigma_{\mathrm{E}}^{\mathrm{fit}}\right)^{2}-\left(\sigma_{\mathrm{E}}^{\mathrm{set}}\right)^{2}} .
$$

In the following, we show that there is a linear relation between the correction $\sigma_{\mathrm{E}, \epsilon_{x}}$ and $\epsilon_{x}$, within a constrained parameter space.

The parameter space $\left(\epsilon_{x}, \sigma_{\mathrm{E}}^{\text {set }}\right)$ used in finding the emittance correction expression is shown in Fig. 8 as a shaded area. Each point in the shaded area represents a pair of $\left(\epsilon_{x}, \sigma_{\mathrm{E}}^{\text {set }}\right)$ which is used to generate a simulated spectrum while other beam and optical klystron parameters from Table I are fixed. This area, defined by $a_{x} \in[1,4.5]$ and $4.00 \times 10^{-4} \leq \sigma_{\mathrm{E}}^{\text {set }} \leq 1.60 \times 10^{-3}$, is chosen using two criteria. The first one, via the choice of $a_{x}$, is that the beam emittance not be too small compared with the energy spread, to ensure that the emittance induced spectrum broadening is significant enough to measure. The second is that the energy spread be in a range which corresponds to a range of horizontal emittance values of interest, in this case from 3 to $50 \mathrm{~nm}$. After the emittance correction expression is determined, it can be applied more broadly beyond the shaded area including to regions where the emittance effect is not expected to be significant.

The energy spread correction for the emittance effect $\sigma_{\mathrm{E}, \epsilon_{x}}$ is well approximated by a linear function of the emittance (Fig. 9),

$$
\sigma_{\mathrm{E}, \epsilon_{x}}=A \epsilon_{x}+B,
$$




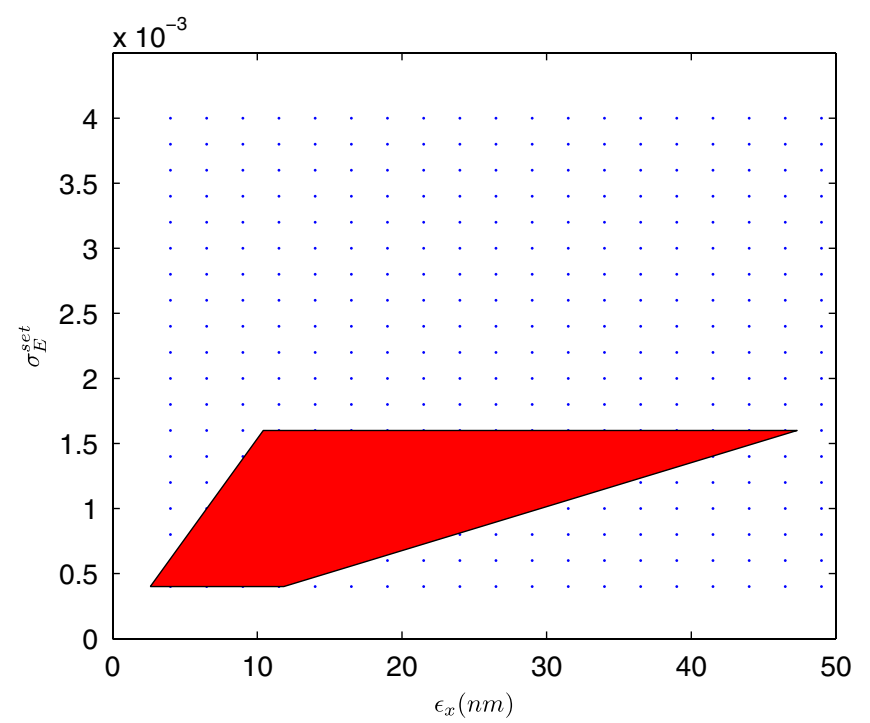

FIG. 8. (Color) Constrained parameter space of $\left(\sigma_{\mathrm{E}}^{\text {set }}, \epsilon_{x}\right)$, the shaded area, is used to determine the emittance correction formula. The shaded area is defined by $a_{x} \in[1,4.5]$ and $4.00 \times$ $10^{-4} \leq \sigma_{\mathrm{E}}^{\text {set }} \leq 1.60 \times 10^{-3}$.

where $A$ and $B$ are constants for a particular experimental setup. They can be found by least-squares fitting Eq. (25) to points simulated using beam parameters $\left(\epsilon_{x}, \sigma_{\mathrm{E}}^{\text {set }}\right)$ in the shaded area in Fig. 8. Figure 9 shows this linear fit, which yields $A=2.03 \times 10^{4}$ and $B=1.01 \times 10^{-4}$. Consequently, the emittance effect $\sigma_{\mathrm{E}, \epsilon_{x}}$ can be calculated accordingly using Eq. (25) for different values of $\epsilon_{x}$. Using these coefficients, the corrected energy spread can be determined using the expression,

$$
\sigma_{\mathrm{E}}^{\text {corr }}=\sqrt{\left(\sigma_{\mathrm{E}}^{\mathrm{fit}}\right)^{2}-\sigma_{\mathrm{E}, \epsilon_{x}}^{2}}
$$

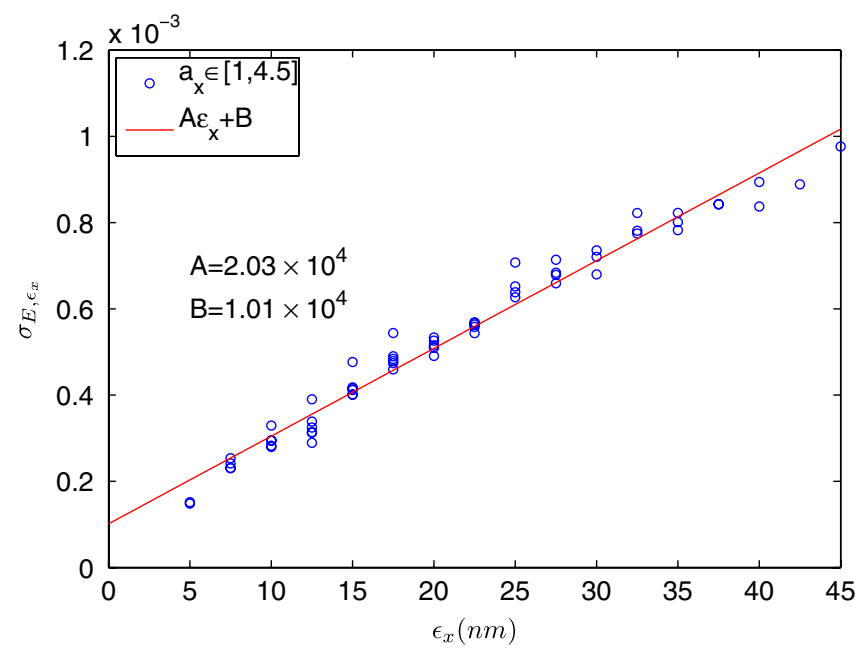

FIG. 9. (Color) Additional spectrum broadening, $\sigma_{\mathrm{E}, \epsilon_{x}}$, as a function of the horizontal electron beam emittance $\epsilon_{x} . \sigma_{\mathrm{E}, \epsilon_{x}}$ is fit to a linear function of $\epsilon_{x}$ and the fitted parameters are listed in the plot.

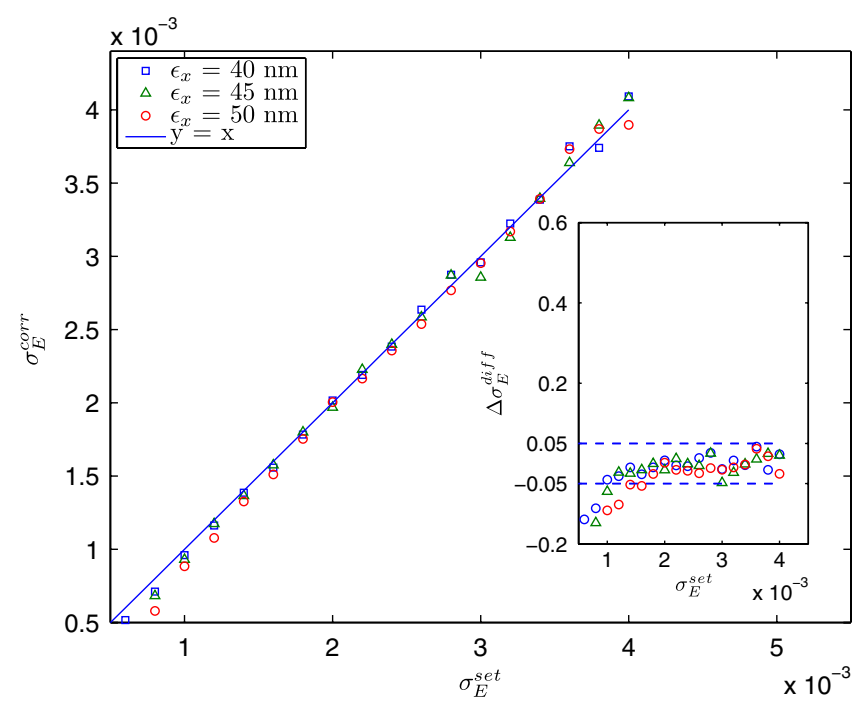

FIG. 10. (Color) Comparison of the emittance-effect corrected energy spread $\sigma_{\mathrm{E}}^{\text {corr }}$ and set value $\sigma_{\mathrm{E}}^{\text {set }}$ for $\epsilon_{x}=40,45,50 \mathrm{~nm}$. The inset shows the relative difference between $\sigma_{\mathrm{E}}^{\text {corr }}$ and $\sigma_{\mathrm{E}}^{\text {set }}$.

Applying the correction scheme of Eq. (26) to the data points plotted in Fig. 7, the fitted energy spread with emittance correction is compared with the set energy spread in Fig. 10. Several observations are in order. First, compared to Fig. 7, the corrected values agree very well with the set values of the energy spread in the entire range of the plot as $\sigma_{\mathrm{E}}^{\text {set }}$ varies from $5.00 \times 10^{-4}$ to $4.00 \times 10^{-3}$. Second, the correction allows us to determine the energy spread with a high degree of accuracy even when the emittance effect is significant. The fitted value is within $\pm 5 \%$ of the set value for $\sigma_{\mathrm{E}}^{\text {set }}>1.00 \times 10^{-3}$. Third, before the correction, the fitted values are consistently higher than the set values; with the correction, the fitted values are scattered around the set values in the region $\sigma_{\mathrm{E}}^{\text {set }}>1.00 \times$ $10^{-3}$, showing no particular statistical bias. Finally, in the region $\sigma_{\mathrm{E}}^{\text {set }}<1.00 \times 10^{-3}$, some overcorrection is observed as the correction reduces the discrepancy from $30 \% \sim 60 \%$ (before correction) to $-5 \% \sim-14 \%$ (after correction). This overcorrection can be significantly reduced if compensation coefficients $A$ and $B$ [Eq. (25)] are determined in the small parameter region of $\left(\epsilon_{x}, \sigma_{\mathrm{E}}^{\text {set }}\right)$.

\section{ENERGY SPREAD MEASUREMENT USING OPTICAL KLYSTRON}

\section{A. Experiment setup and hardware calibration}

At the Duke FEL Laboratory (DFELL), we operate storage ring FELs with several configurations [14]. One is the OK-4 FEL with two planar undulators, and another is the OK-5 FEL with two helical undulators. The electron beam spontaneous radiation spectra from either OK-4 or OK-5 can be used to measure the electron beam energy spread. Figure 11 is a schematic of the experimental layout. For this work all measured spectra are from the OK-4 


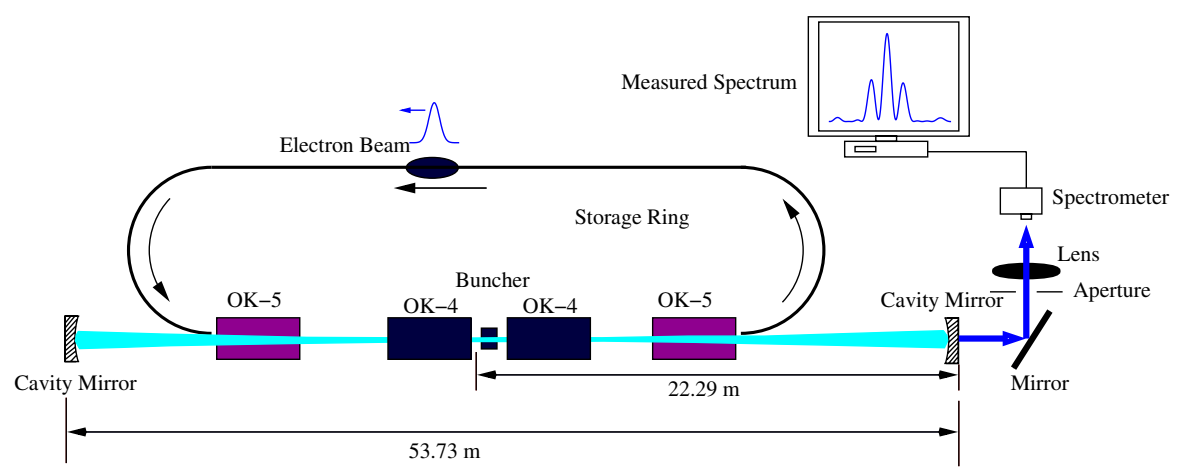

FIG. 11. (Color) A schematic of the experimental setup to measure the electron beam energy spread using a diagnostic optical klystron system on the Duke storage ring.

system. The measured spectra were produced using a single-bunch electron beam at $400 \mathrm{MeV}$. The single-pass spontaneous radiation light is directed to a miniature highresolution fiber optic spectrometer, an Ocean Optics HR4000. The spectrometer has an FWHM optical resolution of $0.11 \mathrm{~nm}$ in the spectral range of 400 to $610 \mathrm{~nm}$ [20]. To produce reliable spectra, the light is directly injected onto the input slit of the spectrometer in the free space without using an optical fiber. The undulator radiation is collimated by a round aperture with a half opening angle of $27 \mu \mathrm{rad}$ before being focused onto the input slit of the spectrometer.

To determine the real spectra intensity, the spectral response of the grating has to be taken into account. The grating efficiency of the spectrometer HR4000 is carefully measured using a technique [21] based on the known undulator radiation spectrum and is described briefly here.

The single undulator spontaneous radiation intensity on the axis as a function of the radiation wavelength is given by

$$
\begin{aligned}
I(\lambda)= & \frac{e^{2} K_{u}^{2} L_{u}^{2}}{8 \epsilon_{0} \gamma_{0}^{2}} \cdot\left[J_{0}(\xi)-J_{1}(\xi)\right]^{2} \cdot \frac{1}{\lambda^{4}} \\
& \times\left[\frac{\sin \left[\pi N_{u}\left(\lambda_{r} / \lambda-1\right)\right]}{\pi N_{u}\left(\lambda_{r} / \lambda-1\right)}\right]^{2} .
\end{aligned}
$$

In this expression, a fixed electron beam energy $\gamma_{0}$ is used. When the electron beam current and energy are fixed, the peak spectrum intensity is proportional to the following quantity determined by the undulator setting:

$$
f_{u}=\frac{K_{u}^{2}\left[J_{0}(\xi)-J_{1}(\xi)\right]^{2}}{2\left(1+\frac{K_{u}^{2}}{2}\right)^{4}}
$$

The relative grating efficiency is the normalized ratio of the measured spectra intensity to the factor $f_{u}$ at a fixed electron beam current for each wavelength of interest. By varying $K_{u}$, we can obtain the efficiency as a function of the wavelength. Figure 12 shows the grating efficiency curve which has a trend of steep increase from 420 to $515 \mathrm{~nm}$. The measured spectra are in the wavelength region from 430 to $470 \mathrm{~nm}$, with an increase of the grating efficiency by a factor of 4.5 . The correction for the grating response is therefore critical for determining the true spectra from the measured ones.

To get the spontaneous radiation out of the FEL optical resonator, the OK-4 spectra are tuned to a wavelength region where the FEL mirror has a good transmission. The second correction on the measured spectra is to compensate for the nonuniform transmission of the FEL mirror as a function of wavelength. The measured FEL mirror transmission curve has been used to rescale the measured spectra.

Figure 13 shows a measured raw spectrum and the spectrum after correction for both the grating efficiency and FEL mirror transmission. At the time when the raw

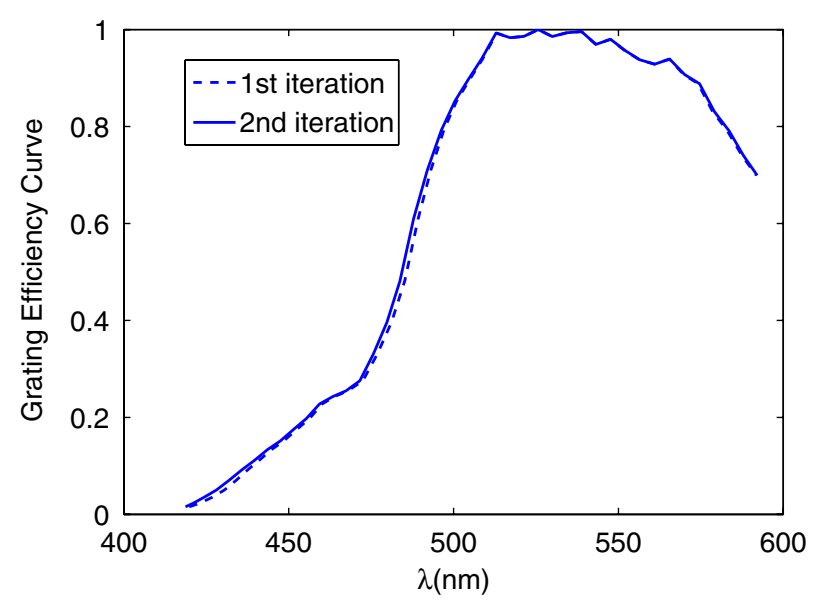

FIG. 12. (Color) The measured grating efficiency curve for the Ocean Optics HR4000 spectrometer. The efficiency curve is calibrated using known spontaneous undulator spectra which are varied to cover the wavelength range of the spectrometer. The efficiency curve converges after a few iterations. The dotted curve is the efficiency curve after the first iteration; and the solid curve is the converged efficiency curve after two iterations. 


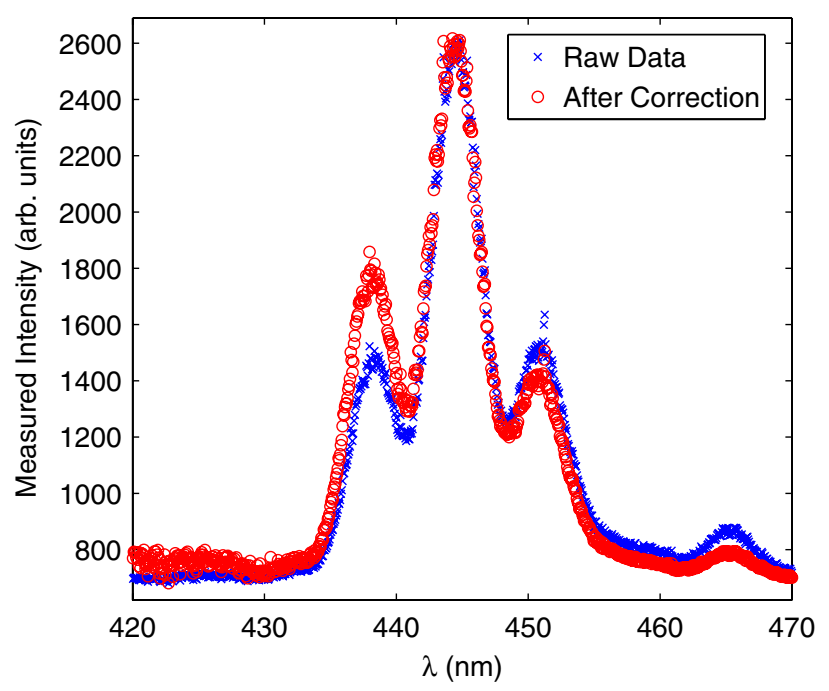

FIG. 13. (Color) A typical measured optical klystron spectrum before and after the correction using the grating efficiency curve. The raw spectrum is represented by blue crosses while the corrected spectrum is shown using red circles.

spectrum was measured, to build in more redundancy in the spectra, the buncher magnet was set to a value so that the raw spectrum appeared to be symmetric with respect to the center peak. After applying corrections, the real spectrum is no longer symmetric. However, this loss of symmetry does not significantly affect the fitting result for the energy spread. Because of the steep change in the grating efficiency curve, the low and high wavelength tails of the raw spectrum settle at different levels. With the correction, the background level at the low and high wavelengths becomes more even. However, the secondary peak associated with the sinc-function is still buried in noise on the shorter wavelength side due to the lower grating efficiency at short wavelengths.

\section{B. Measured energy spread with a large $N_{d}$}

For an optical klystron configuration with a large $N_{d}=$ 31 , a number of radiation spectra have been measured and analyzed. To manipulate the energy spread of the electron beam, the FEL interaction is turned on using the OK-5 system. The FEL interaction can dynamically change the electron beam energy spread. In the Duke storage ring, the FEL interaction can be manipulated via changing the synchronization condition between the electron beam and FEL beam by fine-tuning the rf frequency gradually. However, the degree of the FEL interaction was controlled to make sure that electron beam energy spread did not grow beyond the range which the optical klystron with a large $N_{d}$ is suited for. Figure 14 shows a series of measured and corrected spectra and fitted curves using the G-H method and SES method. The fitted curves by the G-H method match the measured spectra well. However, the SES method shows an increased level of discrepancy in the
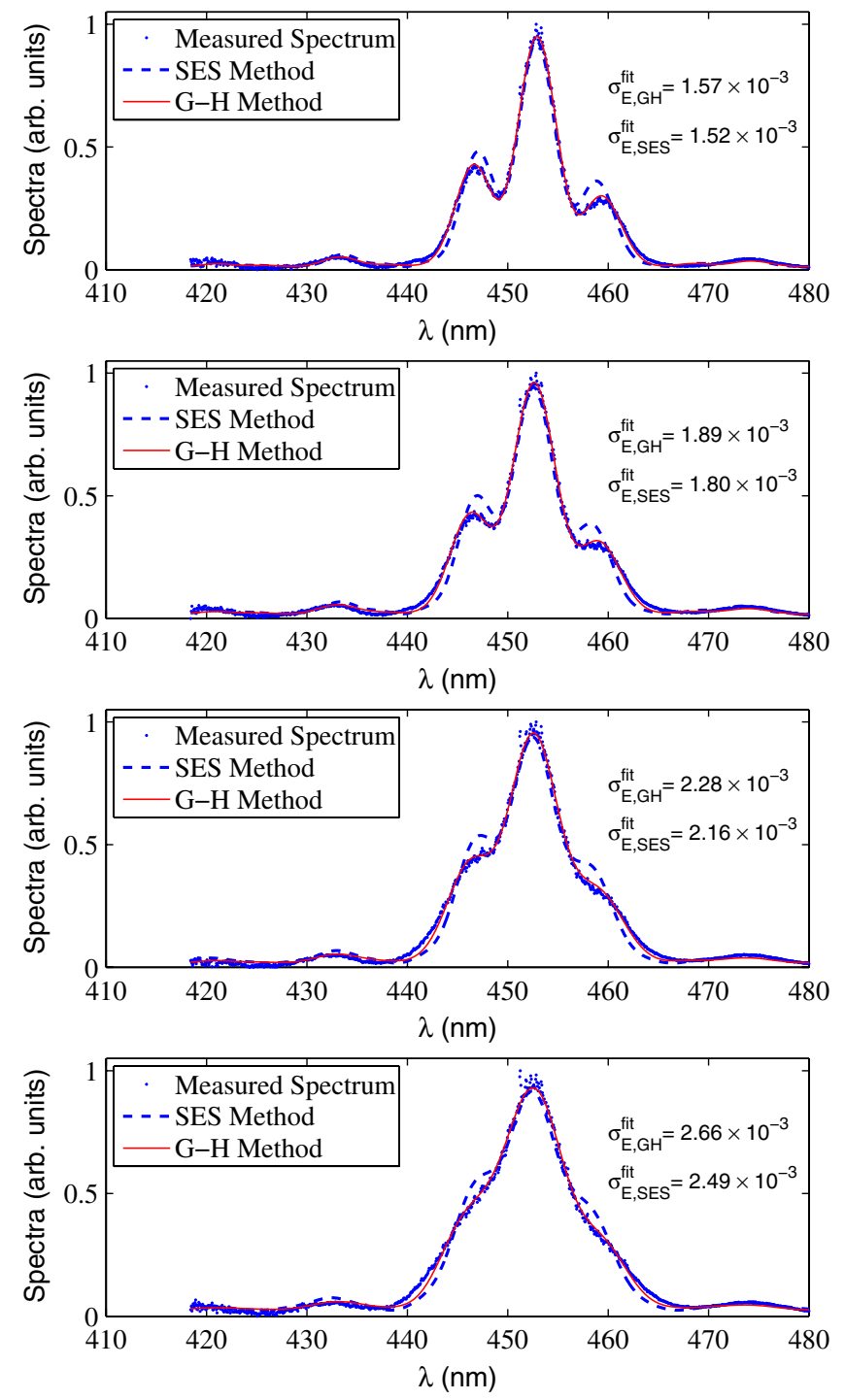

FIG. 14. (Color) Measured optical klystron spectra with a varying energy spread and a high buncher setting $N_{d}=31$. The electron beam energy is $400 \mathrm{MeV}$ and the single-bunch beam current is about $15 \mathrm{~mA}$. The FEL interaction is adjusted to increase the energy spread of the beam. Both the G-H method and the SES method are used to fit the measured spectra. The fitted values of the energy spread using both methods are listed in the plot. In each subplot, the background of the measured spectrum, $h$ in Eq. (6), has been subtracted.

fitting as the energy spread increases. The fitted values of the energy spread using the G-H method are shown in the first column of Table II. All the measured energy spread values are less than $\sigma_{L}\left(\approx 2.85 \times 10^{-3}\right)$, a region where a large $N_{d}$ optical klystron configuration works the best. The statistical uncertainty of the energy spread fitted value using the G-H method is typically less than $\pm 1 \%$. The SES method is also used to fit the energy spread. Compared with the G-H method, the SES method produces relatively poor fitting and it consistently underestimates the energy spread by $3 \%$ to $7 \%$ as shown in Table II. 
TABLE II. Fitted values of the energy spread with $N_{d}=31.0$ (Fig. 14) and the effect of emittance correction. The corrected energy spread is given by $\sigma_{\mathrm{E}, \mathrm{GH}}^{\mathrm{corr}}$.

\begin{tabular}{cccc}
\hline \hline $\begin{array}{c}\text { G-H fit } \\
\sigma_{\mathrm{E}, \mathrm{GH}}^{\mathrm{fit}}\left(\times 10^{-3}\right)\end{array}$ & $\begin{array}{c}\text { SES fit } \\
\text { fit }\end{array}\left(\times 10^{-3}\right)$ & $\sigma_{\mathrm{E}, \mathrm{GH}}^{\mathrm{corr}}\left(\times 10^{-3}\right)$ & $\left(\sigma_{\mathrm{E}, \mathrm{GH}}^{\mathrm{fit}}-\sigma_{\mathrm{E}, \mathrm{GH}}^{\mathrm{corr}}\right) /\left(\sigma_{\mathrm{E}, \mathrm{GH}}^{\text {corr }}\right)$ \\
\hline $1.57 \pm 0.005$ & $1.52 \pm 0.011$ & 1.49 & $5.4 \%$ \\
$1.89 \pm 0.005$ & $1.80 \pm 0.013$ & 1.82 & $3.9 \%$ \\
$2.28 \pm 0.006$ & $2.16 \pm 0.016$ & 2.23 & $2.2 \%$ \\
$2.66 \pm 0.008$ & $2.49 \pm 0.021$ & 2.62 & $1.5 \%$ \\
\hline \hline
\end{tabular}

\section{Emittance correction to experimental data}

To compensate for the angular spread effect, the beam emittance is measured using the beam image at a synchrotron radiation port which collects electron beam radiation from a dipole magnet. The transverse beam image shown in Fig. 15 is taken by a CCD camera with pixel size of 4.6 $\mu \mathrm{m}$. At DFELL, the eta function at this location is small; the contribution of the electron beam energy spread to the beam size is negligible. With the measured beam size and beta function $\beta_{x}=1.3 \mathrm{~m}$ at the measurement location, the emittance can be determined to be $\epsilon_{x}=19 \mathrm{~nm}$.

Emittance correction is applied to the spectra shown in Fig. 14. The emittance correction model of Sec. V predicts an energy spread contribution due to $19 \mathrm{~nm}$ horizontal emittance is $\sigma_{\mathrm{E}, \epsilon_{x}}^{\mathrm{fit}}=4.9 \times 10^{-4}$. Applying the emittance correction, the corrected energy spread is shown in Table II. The emittance correction reduces the fitted energy spread values by $2 \%-5 \%$, which is on the same order of the overall fitting uncertainty. This is strong evidence that in these measurements the emittance effect is not important. In fact, for most of our storage ring operation configurations, the emittance effect in the energy spread measurement is negligible. The emittance correction is useful if the storage ring is operated at lower energy region with a smaller energy spread.

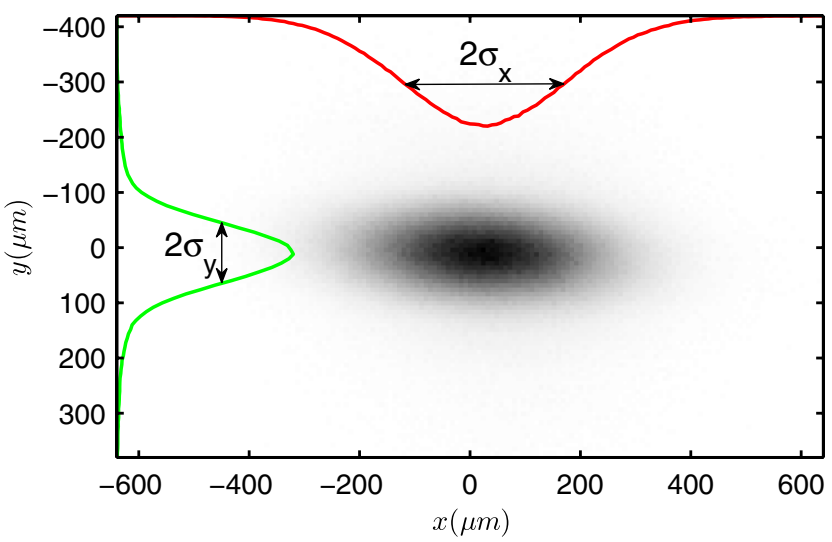

FIG. 15. (Color) The transverse beam image measured at the synchrotron radiation port. The electron beam energy is $400 \mathrm{MeV}$ and single-bunch beam current is $15 \mathrm{~mA}$. Horizontal and vertical projections of the beam transverse profile are also shown. A Gaussian fit of the horizontal beam distribution yields $\sigma_{x}=0.16 \mathrm{~mm}$.

\section{Measured energy spread with a small $N_{d}$}

By intensifying the FEL interaction, the electron beam energy spread can be further increased to a level $\left(\sigma_{E}>\right.$ $\sigma_{L}$ ) at which the broadened undulator spectrum can be measured using an optical klystron with a small $N_{d}$. Figure 16 shows some of the measured spectra with $N_{d} \sim$ 1. Using the $\mathrm{G}-\mathrm{H}$ method, the electron energy spread is found to be $3.09 \times 10^{-3}$ to $3.56 \times 10^{-3}$ as the FEL interaction is slightly increased, with an uncertainty of $\pm 1 \%$. At
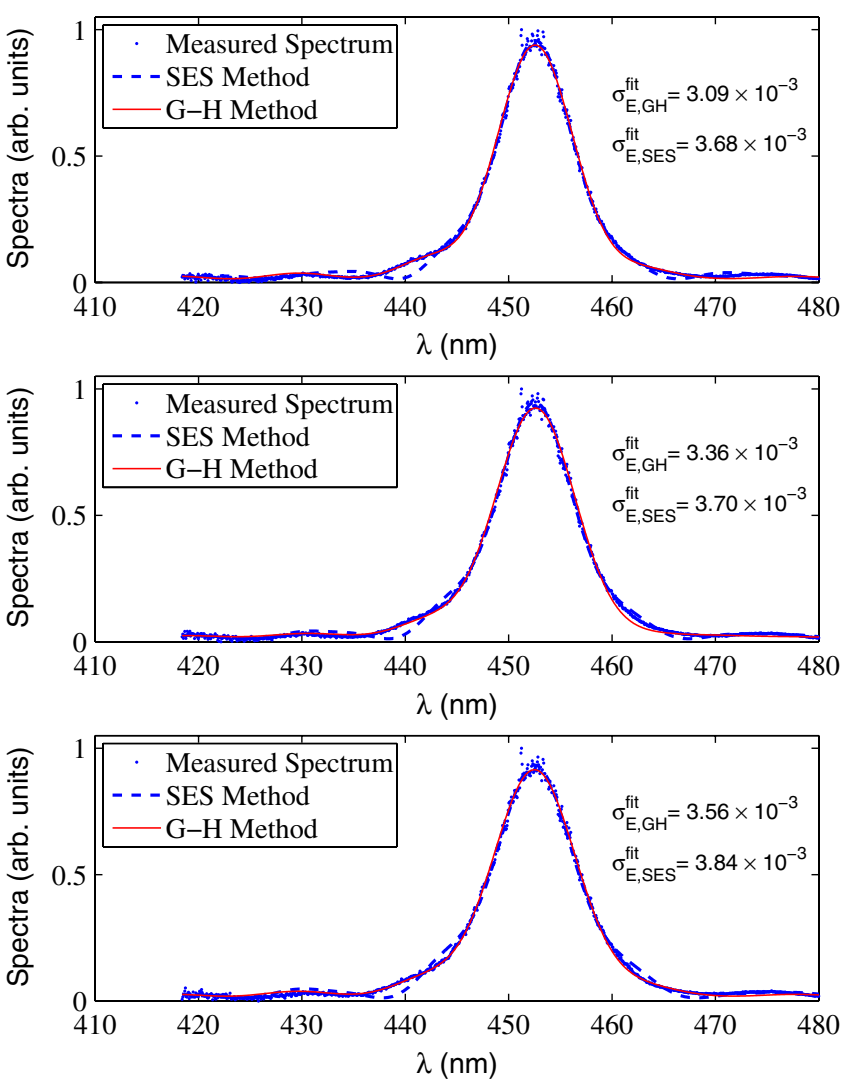

FIG. 16. (Color) Measured optical klystron spectra with an increased energy spread and a low buncher setting $N_{d}=1.09$. The electron beam energy is $400 \mathrm{MeV}$ and the single-bunch beam current is about $15 \mathrm{~mA}$. Compared to Fig. 14, the FEL interaction is increased to produce a larger energy spread. Both the G-H method and the SES method are used to fit the measured spectra. The fitted values of the energy spread using both methods are listed in the plot. In each subplot, the background of the measured spectrum has been subtracted. 
TABLE III. Fitted values of the energy spread with $N_{d}=1.09$ (Fig. 16) and relative difference of the two fitting methods.

\begin{tabular}{ccc}
\hline \hline G-H fit & SES fit & \\
$\sigma_{\mathrm{E}, \mathrm{GH}}^{\mathrm{fit}}\left(\times 10^{-3}\right)$ & $\sigma_{\mathrm{E}, \mathrm{SES}}^{\mathrm{fit}}\left(\times 10^{-3}\right)$ & $\left(\sigma_{\mathrm{E}, \mathrm{SES}}^{\mathrm{fit}}-\sigma_{\mathrm{E}, \mathrm{GH}}^{\mathrm{fit}}\right) /\left(\sigma_{\mathrm{E}, \mathrm{GH}}^{\mathrm{fit}}\right)$ \\
\hline $3.09 \pm 0.010$ & $3.68 \pm 0.021$ & $19 \%$ \\
$3.35 \pm 0.011$ & $3.70 \pm 0.017$ & $10 \%$ \\
$3.56 \pm 0.010$ & $3.84 \pm 0.018$ & $8 \%$ \\
\hline \hline
\end{tabular}

this relatively large energy spread, the emittance correction is no longer needed. The SES method is also used, which produces relatively poor fit as compared with the G-H method. The fitted energy spread using the SES method is consistently higher than those found using the G-H method by $8 \%-19 \%$ (see Table III). Unlike the case with a smaller energy spread and a large $N_{d}$ (Table II), the SES method overestimates the electron beam energy spread in this region.

The overall uncertainty of the relative energy spread measurement is dominated by systematic uncertainties. The most important contributions come from three areas. The first one is the misalignment of the collimating aperture before the spectrometer (see Fig. 11) with a systematic error contribution of less than $0.5 \%$. The second source is the calibration error of the grating response and mirror transmission. This can contribute to a systematic uncertainty of $0.5 \%$ to $2 \%$. The third one is the model dependent systematic uncertainty (the G-H method and related fitting method) at a level of about $2 \%$.

\section{E. Dynamic range of energy spread measurement using optical klystron}

As a diagnostic system to measure electron beam energy spread, an optical klystron is expected to be useful for a wide range of values of the energy spread spanning 2 orders of magnitude, for example, $\sigma_{E}=1 \times 10^{-4}$ to $1 \times$ $10^{-2}$, depending on the number of periods of the undulators in the system. Electron beams with such a wide range of energy spread are impossible to produce in one particular storage ring. To study the dynamic range of the energy spread measurement technique using the optical klystron radiation, we developed various operation configurations of the Duke storage ring. As part of the study of the onset of microwave instability, low energy beams $(E=280 \mathrm{MeV})$ with very small currents were produced [15]. Figure 17 shows a measured optical klystron spectrum for a beam with a $45 \mu \mathrm{A}$ of current. The buncher magnet is set to a high value $\left(N_{d} \approx 56\right)$ to enhance the intensity modulation. Using the G-H method, the energy spread is found to be $\sigma_{\mathrm{E}}^{\mathrm{fit}}=(6.00 \pm 0.015) \times 10^{-4}$. No emittance correction is performed as the emittance induced broadening is expected to be small for this low current beam. To reach the highenergy spread region, controlled FEL interaction is used. Figure 18 shows a case with a $40 \mathrm{~mA}$ single-bunch current

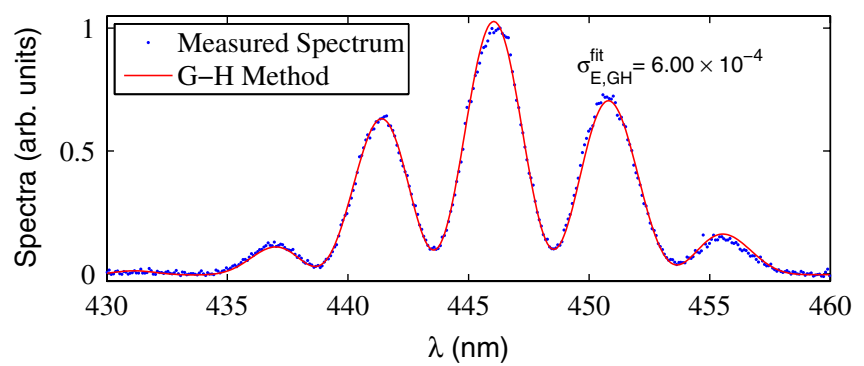

FIG. 17. (Color) Measured optical klystron spectrum with a very small energy spread. The electron beam energy is $280 \mathrm{MeV}$ and the single-bunch beam current is $0.045 \mathrm{~mA}$. To produce substantial spectral intensity modulation, the buncher magnet is powered with a large current with $N_{d} \approx 56$. The G-H method is used to find the energy spread of the beam, $\sigma_{\mathrm{E}}^{\mathrm{fit}}=(6.00 \pm$ $0.015) \times 10^{-4}$. The background of the measured spectrum has been subtracted.

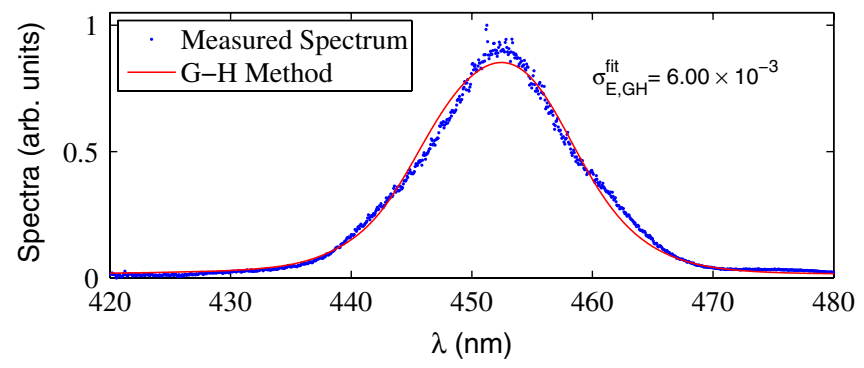

FIG. 18. (Color) Measured optical klystron spectrum with a very large energy spread. The electron beam energy is $400 \mathrm{MeV}$ and the single-bunch beam current is $40 \mathrm{~mA}$. The FEL interaction is turned on to increase the energy spread. The buncher magnet is powered with a small current with $N_{d}=1.06$. The G-H method is used to find the energy spread of the beam, $\sigma_{\mathrm{E}}^{\mathrm{fit}}=(6.00 \pm$ $0.029) \times 10^{-3}$. The background of the measured spectrum has been subtracted.

at $400 \mathrm{MeV}$. The measured spectrum is fitted using both the G-H method and the simple Gaussian fit, and the energy spread is found to be $\sigma_{\mathrm{E}, \mathrm{GH}}^{\mathrm{fit}}=(6.00 \pm 0.029) \times 10^{-3}$, and $\sigma_{\mathrm{E}, \text { Gaussian }}^{\text {fit }}=(6.28 \pm 0.080) \times 10^{-3}$, respectively. Using various configurations of the electron beams at the Duke storage ring, we have demonstrated the measurement of the energy spread from $6 \times 10^{-4}$ to $6 \times 10^{-3}$ using the optical klystron radiation with an overall uncertainty of less than $5 \%$.

\section{CONCLUSION}

In this work, we have presented a noninvasive, versatile, and highly accurate method to measure the energy spread of the electron beam in a storage ring using optical klystron radiation. This work significantly extends existing methods of energy spread measurement via optical klystron to regimes where the electron beam has a large energy spread and/or a large emittance. 
The optical klystron can be configured with either a high buncher setting to enhance the spectral modulation when the electron beam energy spread is small, or with a low buncher setting to increase the sensitivity to the spectral broadening induced by the energy spread. We have developed a novel numerical model for the optical klystron spectrum, using Gauss-Hermite expansion, which treats both spectral modulation and broadening on an equal footing. With this new model and associated fitting methods, optical klystron radiation can be used to measure the energy spread of an electron beam across a wide range, typically from $10^{-4}$ to $10^{-2}$ (the relative energy spread). Spectral broadening due to angular spread of the electron beam was also studied in detail using simulated spectra, and an effective compensation scheme for the emittance effect described. Using optical klystron radiation, we have successfully measured the relative energy spread of the electron beam in the Duke storage ring from $6.0 \times 10^{-4}$ to $6.0 \times 10^{-3}$, with an overall uncertainty of less than $5 \%$.

This work demonstrates that the optical klystron is a powerful diagnostic for highly accurate measurement of the energy spread of the electron beam in a storage ring. This technique may also be applicable to other advanced accelerators such as high-energy linear accelerators and energy recovery linacs (ERLs) when a noninvasive, highly accurate energy spread measurement is desired. For example, the energy spread is a very important quantity for $\mathrm{x}$ ray free-electron lasers (XFELs). Because of its extremely small value, the measurement of the slice energy spread is rather challenging. For Linac Coherent Light Source (LCLS), the world's first operating XFEL [22], the relative slice energy spread is estimated to be below $10^{-4}$. Conventionally, the energy spread is measured using the electron beam size at an optical transition radiation (OTR) screen in a dispersive region. However, a strong coherent optical transition radiation effect can dominate the beam image, making energy spread measurement impossible [23]. At LCLS, a wire scanner method has been used to map out the electron beam size by performing multiple scans. This method can only measure the average energy spread with many beam shots even after proper correction of beam jitters. For XFELs, optical klystron radiation can be considered as a useful diagnostic for single-pass energy spread measurement. The noninvasive nature of this method also makes it attractive for ERLs in which an invasive technique using a screen may not be feasible due to a very large amount of beam power.

\section{ACKNOWLEDGMENTS}

We would like to thank Juhao Wu at SLAC for insightful discussions on using the optical klystron for measuring the single-pass electron beam energy spread for x-ray FELs such as LCLS. We are grateful to Vladimir Litvinenko at BNL for sharing his in-depth knowledge of early measure- ments of electron beam energy spread using an optical klystron at Novosibirsk, Russia. This work is supported in part by the U.S. Department of Energy, Office of Nuclear Physics under Grant No. DE-FG02-97ER41033.

[1] N. A. Vinokurov and A. N. Skrinsky, BINP Report No. 7767, Novosibirsk, Russia, 1977.

[2] N.A. Vinokurov and A. N. Skrinsky, Relativistic Microwave Electronics (Gorky Polytechnic Institute, Gorky, 1981), p. 204.

[3] A. Renieri, Nuovo Cimento B 53, 160 (1979).

[4] H. R. Weller et al., Prog. Part. Nucl. Phys. 62, 257 (2009).

[5] J. Seeman, Energy Measurement with Electron Beams in Handbook of Accelerator Physics and Engineering, edited by W. Chao and M. Tigner (World Scientific, Singapore, 1999).

[6] E. Tarazona et al., Rev. Sci. Instrum. 67, 3368 (1996).

[7] B.X. Yang and J. J. Xu, in Proceedings of the Particle Accelerator Conference, Chicago, IL, 2001 (IEEE, New York, 2001), p. 2338.

[8] B. X. Yang and A. H. Lumpkin, in Proceedings of the 21st Particle Accelerator Conference, Knoxville, TN, 2005 (IEEE, Piscataway, NJ, 2005), p. 74.

[9] R. Klein et al., Nucl. Instrum. Methods Phys. Res., Sect. A 384, 293 (1997).

[10] C. Sun, J. Li, G. Rusev, A. P. Tonchev, and Y. K. Wu, Phys. Rev. ST Accel. Beams 12, 062801 (2009).

[11] N. A. Vinokurov and A. N. Skrinsky, BINP Report No. $77-$ 59, Novosibirsk, Russia, 1977.

[12] G. A. Kornyukhin et al., Nucl. Instrum. Methods Phys. Res., Sect. A 237, 281 (1985).

[13] P. Elleaume, J. Phys. 44, 333 (1983).

[14] Y. K. Wu, N. A. Vinokurov, S. Mikhailov, J. Li, and V. Popov, Phys. Rev. Lett. 96, 224801 (2006).

[15] S. Huang, J. Li, and Y. K. Wu, in Proceedings of the 2007 Particle Accelerator Conference, Albuquerque, New Mexico (IEEE, Albuquerque, New Mexico, 2007), p. 4045.

[16] K.-J. Kim, AIP Conf. Proc. 185, 565 (1988).

[17] B. Jia, Master's thesis, Duke University. 2009.

[18] M. Abramowitz and I. A. Stegun, Handbook of Mathematical Functions 10th Printing with Corrections (Dover, New York, 1972), ISBN 978-0-486-61272-0.

[19] P. J. Davis and P. Rabinowitz, Methods of Numerical Integration (Dover, New York, 1975).

[20] High-Resolution Fiber Optic Spectrometers HR4000 Series. Installation and Operation Manual Document Number 210-00000-000-02-0908, http://oceanoptics.com (2009).

[21] S. Huang, Ph.D. dissertation, Beijing University, 2009.

[22] P. Emma, in Proceedings of the 23rd Particle Accelerator Conference, Vancouver, Canada, 2009 (IEEE, Piscataway, NJ, 2009).

[23] H. Loos et al., in Proceedings of the 30th International Free Electron Laser Conference (FEL'08) (JACoW, Gyeongju, Korea, 2008), p. 485. 